\title{
Les appels à projet franco-allemands ANR/DFG en sciences sociales et humaines
}

\section{Pierre Monnet}

\section{(2) OpenEdition}

\section{Journals}

Édition électronique

URL : http://journals.openedition.org/ifha/426

DOI : 10.4000/ifha.426

ISSN : 2198-8943

\section{Éditeur}

IFRA - Institut franco-allemand (sciences historiques et sociales)

\section{Édition imprimée}

Date de publication : 30 septembre 2012

Pagination : 133-136

ISSN : 2190-0078

\section{Référence électronique}

Pierre Monnet, "Les appels à projet franco-allemands ANR/DFG en sciences sociales et humaines », Revue de l'IFHA [En ligne], 4 | 2012, mis en ligne le 14 février 2013, consulté le 02 mai 2019. URL http://journals.openedition.org/ifha/426 ; DOI : 10.4000/ifha.426

Ce document a été généré automatiquement le 2 mai 2019.

(CIFHA 


\title{
Les appels à projet franco-allemands ANR/DFG en sciences sociales et humaines
}

\author{
Pierre Monnet
}

1 Depuis 2007, l'Agence nationale de la recherche (ANR) et la Deutsche Forschungsgemeinschaft (DFG) se sont associées pour lancer un appel à projets commun afin de développer les liens scientifiques entre équipes de recherche françaises et allemandes dans toutes les disciplines des sciences sociales et humaines. Ces projets de recherche, financés en règle générale à part égale par les deux agences de moyens à hauteur de 150.000 à 300.000 euros selon les projets pour une durée de 3 ans et plus spécialement ouverts à l'intégration des post-doctorants depuis 2010, ont pris acte dès leur création des liens déjà serrés que des universités, laboratoires et centres de recherche des deux pays avaient noués dans le champ des humanités. Un bilan des quatre éditions qui se sont déroulées de 2007 à 2010 a montré que, parmi les disciplines concernées, les projets soumis (284 en 4 ans) et retenus (79 en 4 ans, soit un taux de réussite d'environ $27 \%$ ) provenant de l'histoire et de l'histoire de l'art avec 13 projets financés et de l'archéologie avec 11 projets financés (soit 24 en tout, autrement dit le tiers du total) constituaient avec la linguistique (12 projets financés) et l'économie ( 9 projets financés) le premier secteur privilégié par ce dispositif.

2 Parmi les 18 projets retenus en 2011, 6 relèvent de nouveau des sciences historiques, confirmant la place occupée par ce domaine lors des campagnes précédentes. Le succès remporté par les dossiers présentés par les historiens s'explique à la fois par le rôle traditionnel et historique que l'histoire et l'écriture de l'histoire ont tenu depuis le XIXe siècle dans le dialogue entre les communautés scientifiques française et allemande ainsi que dans la formation des idées et des préjugés portés sur l'autre, mais également par la nature des programmes présentés, en particulier en matière d'édition électronique, de corpus de sources et de confection de bases de données. Il est certain que l'existence de centres français et allemands de recherche en histoire de part et d'autre du Rhin, entre l'IFHA de Francfort et le Centre Marc Bloch de Berlin et le CIERA et l'Institut Historique 
Allemand de Paris, a également contribué à la forte représentation des historiens au sein du programme.

3 À ce titre, l'IFHA trouve un triple intérêt à se faire l'écho de ce dispositif dans les colonnes de la Revue. Tout d'abord, cet appel à projets fait émerger des thématiques nouvelles dans le champ de la coopération franco-allemande en matière historique, dont les retombées ont des conséquences directes et indirectes sur la programmation scientifique du centre ainsi qu'en termes de formation de jeunes chercheurs. De surcroît, des moyens supplémentaires sont apportés au sein d'un réseau de recherche animé par l'IFHA et ses partenaires. Ensuite, des équipes et des chercheurs autrefois passés par l'IFHA ou ayant bénéficié de son soutien se trouvent engagés dans les projets financés : deux des membres du conseil scientifique de l'IFHA sont porteurs d'un projet (Jean Mondot, université de Bordeaux, pour « Gallotropisme et modèles civilisationnels dans l'espace germanophone (1660-1789)» depuis 2010 et Christophe Charle, université de Paris I, pour «La transculturalité des espaces nationaux. Processus de traduction, figures de médiation et effets socioculturels des transferts littéraires en Europe (1750-1900) » depuis 2011), tandis que d'anciens boursiers ou membres de la MHFA et de l'IFHA sont aujourd'hui impliqués dans des thématiques communes, ainsi de Patrice Veit, ancien directeur de la MHFA avec "Les usages religieux du livre à l'époque moderne. Une comparaison transculturelle et interconfessionnelle (France-Empire)» (2007); Philippe Depreux, ancien chercheur pensionnaire de la MHFA avec «La productivité d'une crise : le règne de Louis le Pieux (814-840) et la transformation de l'empire carolingien » (2007); Geneviève BührerThierry, ancienne boursière de courte durée de la MHFA avec «Espace et politique: perceptions et pratiques dans les royaumes francs et post-carolingiens du IXe au XIe siècle » (2009) ; Elsa Kammerer, ancienne boursière de courte durée de la MHFA, avec "Dynamique des langues vernaculaires dans l'Europe de la Renaissance. Acteurs et lieux " (2009) ; Bénédicte Savoy, ancienne boursière de courte durée de la MHFA, avec «Formations artistiques transnationales entre la France et l'Allemagne, 1789-1870 " (2009 et 2011) ; Christine Lebeau et Jakob Vogel, anciens boursiers de courte durée de la MHFA, avec « Localisation et circulation des savoirs d'État en Europe, 1750-1850 » (2010). Enfin, l'IFHA est partie prenante de l'un des projets soutenus dès 2007 et renouvelé en 2010, la revue électronique franco-allemande en sciences sociales et humaines Trivium portée par la Maison des Sciences de l'Homme (Hinnerk Bruhns et Falk Bretschneider) et l'Institut historique allemand de Paris (Gudrun Gersmann) : un numéro spécial sur le Saint-Empire moderne à paraître en 2012 est en effet présenté par l'IFHA.

Il convient de relever que les thématiques financées depuis 2007 dans le cadre de cet appel offrent un aperçu des champs historiques actuellement en plein renouvellement de part et d'autre du Rhin. Dès 2007 puis en 2008, trois champs d'intenses échanges scientifiques franco-allemands se détachaient dans une histoire de la longue durée, abstraction faite de l'archéologie : l'histoire des pratiques et des cultures religieuses à l'époque moderne, le transfert des idées, des méthodes et des philosophies en sciences sociales et humaines aux XIXe et XXe siècles, la construction d'entités politicoterritoriales à l'échelle européenne depuis le Moyen Âge. Quant aux thématiques d'une histoire du temps présent au carrefour de plusieurs disciplines, elles tournent dès 2007 et 2008 autour des questions du risque, de l'immigration, du travail et de la santé. La campagne 2009 ajoute à cette palette des interrogations liées au genre, au langage et à la musique. Il est de même intéressant de souligner que du point de vue méthodologique, l'histoire croisée, comparée et transnationale caractérise bon nombre de ces projets : sur 
les 18 dossiers financés entre 2007 et 2011 relevant des sciences historiques, trois portent le prédicat « transnational », quatre celui de " frontalier », deux celui de " transculturel », deux ceux de l'échange et du transfert, soit onze au total qui affichent explicitement dans leur démarche l'approche circulatoire et connectée. Quant au terrain d'études, une évolution remarquable se dessine depuis 2007. En effet, à cette date, 7 des 8 projets soutenus dans le champ de l'histoire ou de la société contemporaine relevaient explicitement d'un espace géographique proprement franco-allemand. Dès 2008 en revanche émergent des objets ou lieux de recherche franco-allemands de dimension européenne ou extra-européenne, tel le thomisme au Moyen Âge ou le royaume arabe de Petra. Cette tendance se confirme et s'amplifie en 2009 avec le Caucase protohistorique, les villes antiques de Syrie, les langues de l'Europe renaissante ou les musiciens de l'Europe baroque. En 2010, avec un champ commun de recherche sur Alexandrie d'Égypte, sur l'histoire des savoirs médicaux ou des savoirs d'État en Europe moderne, sur la HauteMésopotamie antique, sur les traditions religieuses en Asie du Sud-Est, l'on voit bien que c'est davantage le regard que l'espace de l'approche scientifique qui devient francoallemand, un glissement que confirme l'édition 2011 avec l'étude de la lettre dans l'Occident latin, les territoires du Pérou ancien, la vallée de l'Indus protohistorique, le Tibet des XVIIe-XXe siècles ou les espaces socioculturels européens entre 1750 et 1900.

5 Afin de mieux détailler et concrétiser le travail, les résultats, les méthodes et la plus-value de ces projets de recherche franco-allemands, la Revue de l'IFHA a souhaité publier le retour d'expérience porté sur deux dossiers, le premier qui avait démarré en 2007 et est aujourd'hui achevé, celui qu'ont conjointement conduit Patrice Veit (CNRS/Centre Marc Bloch de Berlin) et Thomas Kaufmann (université de Göttingen) autour des «Usages religieux du livre à l'époque moderne " (acronyme "Buchpraxis») et celui, encore en cours car entamé en 2009, encadré par Geneviève Bührer-Thierry (université de Paris-Est Marne-la-Vallée) et Steffen Patzold (université de Tübingen) portant sur «Espace et politique : perceptions et pratiques dans les royaumes francs et post-carolingiens du IXe au XIe siècle » (acronyme « Territorium »).

\section{AUTEUR}

\section{PIERRE MONNET}

Pierre Monnet est directeur de l'IFHA. 